\title{
Cuantificación de los recursos materiales consumidos en la ejecución de la cimentación
}

\section{Quantification of material resources consumed during concrete slab construction}

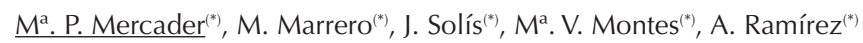

\section{RESUMEN}

\begin{abstract}
La industria de la construcción produce un significativo impacto medioambiental debido a que consume cerca del $40 \%$ de los recursos naturales y genera en torno al $40 \%$ de los residuos. Debe ser función del técnico responsable, entre otros, a través de los procesos de diseño, obtención y suministro de materiales, construcción, uso y demolición final, contribuir a un entorno más sostenible. Es por ello de gran interés identificar y cuantificar los factores que condicionan el impacto ambiental, ya que el único modo en el que se puede hablar de mejoras es a través de la obtención de datos que se puedan medir y contrastar con facilidad. En el presente trabajo se evaluarán estos factores desde la perspectiva de los recursos materiales consumidos durante la fase de construcción de la edificación. Dicha tarea no es sencilla dada la intervención de procesos y agentes muy heterogéneos, desde la extracción y fabricación de materiales hasta la deconstrucción o reciclado.
\end{abstract}

Por último, se presenta un ejemplo práctico en el que se identifican y cuantifican los recursos consumidos en la ejecución de una cimentación. Los resultados de este estudio de campo son ciertamente preocupantes ya que el $100 \%$ de los recursos consumidos constituyen barreras a la sostenibilidad, bien por no ser renovables (90\% de los recursos consumidos) o por la toxicidad que produce su fabricación o puesta en obra para las personas (10\% restante).

\section{3-94}

Palabras Clave: construcción sostenible, identificación y cuantificación de recursos, impacto medioambiental.

\section{SUMMARY}

The construction produces a significant environmental impact; it consumes about $40 \%$ of the natural resources and generates about $40 \%$ of the total waste. Then, the construction technician is responsible, among others, through the design, material selection, construction, usage and final demolition, to reduce the environmental impact. There is a big interest to identify and quantify the factors that define such impact. The only way to improve the system is through an easy measurement and comparison of the resources consumed by different construction methods. The task is not simple because the system involves heterogeneous processing and agents, from the material extraction or fabrication throughout the demolition or recycling.

Finally, a practical example is presented in which the consumed resources are identified and quantified during the foundation construction. The results from the study are worrisome due to the fact that $100 \%$ of the resources are sustainability barriers, for the reason that they are non renewable (90\%) or toxic during production or edification (10\%).

Keywords: sustainable construction, resources identification and quantification, environmental impact. 


\section{INTRODUCCIÓN}

Dentro de las actividades industriales, la actividad constructora, incluida su industria asociada, es la mayor consumidora de recursos naturales tales como madera, minerales, agua y energía. En la Unión Europea, la construcción de edificios consume el $40 \%$ de los materiales, el $40 \%$ de la energía primaria y genera el $40 \%$ de los residuos, teniendo especial responsabilidad en el actual deterioro del medio ambiente la ampliación del parque construido (1). Además, el Plan Nacional de Asignación de Derechos de Emisión 20082012 afirma que el sector doméstico y el de edificación consumen el $20 \%$ del total de la energía final en España, siendo responsables de la emisión de más del $25 \%$ del total de $\mathrm{CO}_{2}$. A estas cifras habría que añadir las emisiones debidas a los procesos de fabricación, transporte y generación eléctrica asociados a la edificación, incluidos actualmente en otros apartados (2).

Evaluar la dimensión medioambiental de los materiales de construcción es intentar calificar y cuantificar el peso de sus impactos durante todo su ciclo de vida, desde la extracción de las materias primas hasta el final del mismo. Por ello es necesario acotar las principales etapas del ciclo de vida de los materiales de construcción tales como: extracción de materias primas o transformación en productos, transporte, construcción, uso, demolición o deconstrucción, y por último, valorización o depósito de residuos. El bucle se cierra si los residuos son reciclados o los materiales reutilizados (Figura 1).

En la primera etapa del ciclo de vida de los materiales de construcción, los costes ecológicos se deben tanto a la extracción de los recursos minerales (canteras, minas, etc.) como a la deposición de los residuos o restos generados (3). Estas afecciones medioambientales abarcan las emisiones tóxicas y el envenenamiento de las aguas subterráneas por parte de los vertederos. Por otra parte, el material fuertemente manipulado y que ha sido sometido a un proceso de fabricación tiene unos efectos medioambientales muy importantes, especialmente desde el punto de vista energético. Entre los materiales con mayor impacto energético encontramos los materiales cerámicos y los metálicos, especialmente el acero. $Y$ entre los de menor impacto, los granulados de piedra, el hormigón prefabricado y la madera (4).

La siguiente etapa la hemos definido como la del transporte de los materiales. Éste genera residuos y contaminación relacionados con el propio medio de transporte como pueden ser chatarra, derrames de aceite y combustible, o residuos peligrosos como las baterías. Su contaminación también está relacionada con la emisión de $\mathrm{CO}_{2}$ a la atmósfera.

Ya en la etapa de construcción del edificio, también se generan residuos y contaminación relacionados con el embalaje, el montaje (corte de piezas, perforaciones, material de agarre, etc.) y la existencia de piezas defectuosas o destruidas durante su manipulación. Tal como establece el principio de jerarquía en la Estrategia comunitaria sobre residuos, resolución del 7 de mayo de 1990, revisada según la nueva Directiva Marco de Residuos de Junio de 2008 para la gestión de los residuos (prevenir, reutilizar, reciclar, valorizar y eliminar), se podrían tomar en esta etapa medidas de prevención (empleo de técnicas constructivas en seco, utilización de elementos prefabricados de gran formato, uso de materiales con certificados ambientales...), de reutilización (chatarra metálica) o incluso reciclado, si habláramos de residuos de origen pétreo.

En la siguiente etapa, la de uso del edificio, al igual que en la previa, estarán presentes las pérdidas de materiales o productos dado el envejecimiento o deterioro que experimentan. Para minimizar la generación de este tipo de residuos es necesario fomentar el mantenimiento y la renovación controlada de los materiales mediante una eficiente identificación del deterioro y su reparación.

Finalmente, tras la demolición o deconstrucción de la edificación, los materiales se convierten en residuos, pudiendo ser reutilizados, reciclados o depositados. En relación con el tratamiento de residuos, es necesario destacar el actual Real Decreto 105/2008, de 1 de febrero, por el que se regula la producción y gestión de los residuos de construcción y demolición (RCD). Una de las motivaciones del nuevo Real Decreto es el establecimiento de modelos de gestión de RCD que permitan el control de los flujos de residuos, de forma que se cierre el ciclo de los materiales, tal como se recoge en la Figura 1. Como objetivos de cabecera del nuevo decreto se establecen prevenir la generación de RCD, fomentar la reutilización 
y el reciclado y asegurar la eliminación con un tratamiento adecuado.

En primer lugar, el decreto define las competencias y responsabilidades de cada uno de los agentes de la edificación. El promotor es considerado el productor de los RCD. El constructor, poseedor de dichos residuos, será responsable de gestionarlos adecuadamente, Ilevándolos a un gestor autorizado que garantice su adecuado tratamiento y valorización. También cabe destacar la exigencia en fase de proyecto de un Estudio de Gestión de Residuos, y en obra de un Plan de Gestión de Residuos. Se contempla también, como mecanismo de control vinculado a la obtención de la licencia de obras, la constitución por parte del productor de una fianza o garantía equivalente que le será reintegrada cuando acredite que los RCD han sido convenientemente gestionados. Con estas medidas el modelo persigue el control de los materiales, de forma que se desincentive el depósito de los residuos en vertedero y se promueva la valorización de los mismos.

En el ciclo de vida antes descrito, una de las etapas que más impacto medioambiental tiene es la extracción o fabricación de los materiales finalmente utilizados en el edificio. Por ello, el primer paso ha sido desarrollar en el presente trabajo una herramienta para la identificación y cuantificación de los recursos consumidos en la construcción de viviendas siguiendo métodos similares a los desarrollados por otros autores, como Albert Cuchí (4) o el estudio de arquitectura SaAS (2). Para ello ha sido necesario identificar el modelo constructivo que más se proyecta en España, cuantificar los materiales que consume y determinar las barreras contra la sostenibilidad. Para cuantificar ha sido necesario clasificar y agrupar los materiales y convertirlos en cantidades que sean fáciles de medir y comparar. Se ha desarrollado un parámetro que transforma la unidad empleada en la medición del proyecto a kilogramos por superficie construida. El trabajo finaliza con una aplicación práctica del modelo, evaluando el impacto medioambiental de la ejecución de la cimentación en tres proyectos reales.

\section{CUANTIFICACIÓN DE RECURSOS}

Para identificar cuáles de los recursos consumidos durante el proceso de ejecución de edificios tienen un mayor impacto medioambiental se ha realizado un trabajo en el que se ha partido de la identificación, cuantificación y análisis de los recursos empleados en el proceso constructivo. A través de las mediciones elaboradas en el

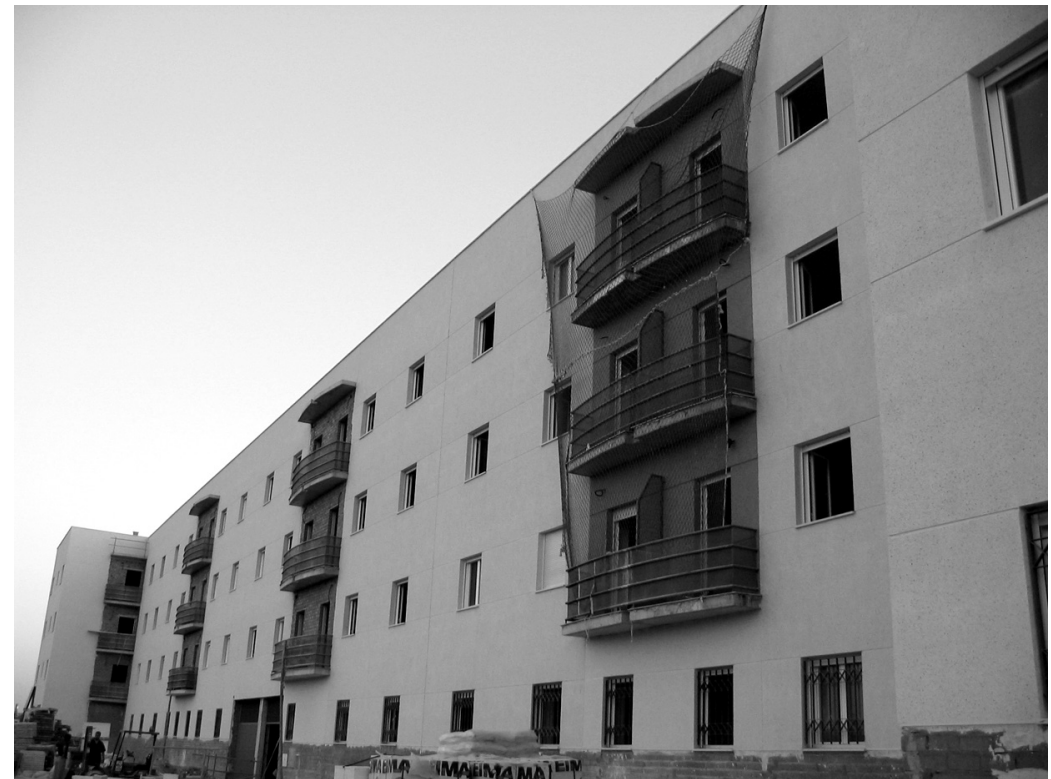

proyecto de ejecución se han calculado los consumos de materiales, pudiendo a partir de ellos deducirse su nivel de impacto medioambiental. El análisis se lleva a cabo según el siguiente proceso:

o Identificación del modelo constructivo convencional.

o Cuantificación de los recursos materiales consumidos en su ejecución.

o Evaluación de su impacto medioambiental.

\subsection{Identificación del Modelo Constructivo Convencional}

Para identificar el modelo representativo de edificación que se realiza a nivel nacional, se ha considerado oportuno acudir, como fuente de información suficientemente amplia y contrastada, a las estadísticas publicadas por el Ministerio de Fomento, Edificación y Vivienda donde se recogen las licencias de obra concedidas por los Ayuntamientos desde el año 1994 hasta el 2003 (5-7). De los datos publicados se deduce que el edificio que más recursos consume está entre 11.000-12.000 $\mathrm{m}^{2}$, y tiene cuatro plantas sobre rasante $y$ una planta bajo rasante destinada a sótano (Figura 2).

El tipo constructivo más numeroso coincide tanto a nivel nacional como en la Comunidad Autónoma de Andalucía, y se caracteriza por:

o Estar constituido por estructura vertical de hormigón armado, estructura horizontal unidireccional, cubierta inclinada, cerramiento exterior cerámico y carpintería exterior de aluminio. 
Tabla 1

Estructura de los proyectos estudiados

\begin{tabular}{|c|c|c|c|}
\hline \multicolumn{4}{|c|}{ ESTRUCTURA DE LOS PROYECTOS ESTUDIADOS } \\
\hline CAPÍTULO & NOMBRE & SUBCAPÍTULO & NOMBRE \\
\hline C.01 & \multicolumn{3}{|l|}{ Movimientos de Tierra } \\
\hline C.02 & \multicolumn{3}{|l|}{ Cimentación } \\
\hline \multirow{3}{*}{ C.03 } & \multirow[t]{3}{*}{ Saneamiento } & С.03.01 & Red colgada \\
\hline & & C.03.02 & Red enterrada \\
\hline & & C.03.03 & Red vertical \\
\hline \multirow{4}{*}{ C.04 } & \multirow[t]{4}{*}{ Estructura } & C.04.01 & Forjados \\
\hline & & C.04.02 & Pilares \\
\hline & & C.04.03 & Muros \\
\hline & & C.04.04 & Escaleras-rampas \\
\hline \multirow{4}{*}{ C.05 } & \multirow[t]{4}{*}{ Cerramientos } & C.05.01 & Hoja exterior \\
\hline & & C.05.02 & Trasdosado \\
\hline & & C.05.03 & Tabiquería interior \\
\hline & & C.05.04 & Otros \\
\hline \multirow{3}{*}{ C.06 } & \multirow[t]{3}{*}{ Cubiertas } & C.06.01 & Transitables \\
\hline & & C.06.02 & No transitables \\
\hline & & C.06.03 & Otros \\
\hline \multirow{4}{*}{ C.07 } & \multirow[t]{4}{*}{ Revestidos horizontales } & C.07.01 & Suelos \\
\hline & & C.07.02 & Techos \\
\hline & & C.07.02 & Escaleras \\
\hline & & C.07.02 & Alfeizares \\
\hline C.08 & Instalaciones & & \\
\hline C.09 & \multicolumn{3}{|c|}{ Carpintería metálica y cerrajería } \\
\hline C. 10 & \multicolumn{3}{|l|}{ Carpintería de madera } \\
\hline C.11 & \multicolumn{3}{|l|}{ Urbanización } \\
\hline C.12 & \multicolumn{3}{|l|}{ Vidrios } \\
\hline C.13 & \multicolumn{3}{|l|}{ Decoración } \\
\hline
\end{tabular}

o Tanto en la Provincia de Sevilla como en Sevilla Capital coincide con el anterior, a excepción de la cubierta donde predomina la cubierta plana en lugar de la inclinada.

o Además, en Sevilla Capital, a partir de los datos obtenidos en la Gerencia Municipal de Urbanismo, en la tipología de cuatro sobre rasante y una planta bajo rasante se constata que la cimentación se resuelve mediante losa armada.

Resumiendo todo lo anterior se elige como modelo constructivo convencional el de planta baja más tres y una planta de sótano bajo rasante, por ser el que más se construye $y$, en consecuencia, el de mayor consumo de recursos materiales.

\subsection{Cuantificación de los recursos materiales consumidos en su ejecución}

Para llevar a cabo la identificación y cuantificación de los recursos consumidos se parte de las mediciones desarrolladas en el proyecto de ejecución de tres proyectos representativos del modelo constructivo convencional (cuatro plantas sobre rasante y una planta bajo rasante destinada a sótano). Los bloques fueron realizados en la barriada de Pino Montano de Sevilla, formando parte de la ejecución de una urbanización de viviendas plurifamiliares de protección oficial, con una superficie comprendida entre $11.000 \mathrm{y}$ $12.000 \mathrm{~m}^{2}$ para cada uno de ellos.

Al iniciar la tarea, se constató que para alcanzar la meta propuesta es necesario conseguir tres objetivos complementarios:

o Dotar al sistema de una estructura que permita identificar con claridad los subsistemas constructivos.

- Dividir a su vez los subsistemas de manera que puedan establecerse comparativos entre distintas obras, estableciendo la relación de los materiales empleados y la proporción en que éstos son utilizados.

o Proporcionar un esquema generalizable a obras de características similares. 
Teniendo en cuenta los puntos antes expuestos, para poder cuantificar los recursos consumidos por cada subsistema constructivo en los edificios estudiados, y poder compararlos, se parte del siguiente procedimiento:

- Estructurar las mediciones de los proyectos en base a una misma división en subsistemas constructivos. Los subsistemas se enumeran en la Tabla 1.

o Obtener los descompuestos de cada partida utilizando la Base de Costes de la Construcción de Andalucía, BCCA (8).

o Obtener los descompuestos de los componentes que no son elaborados en la obra a partir de empresas y laboratorios especializados. Por ejemplo, el caso de los hormigones suministrados cuya descomposición en elementos básicos, tales como cemento, agua, arena, grava y aditivos se ha llevado a cabo según la dosificación del hormigón que más se emplea en España.

o Analizar las mediciones usando un programa informático de presupuestos y mediciones.

- Aplicar un factor de relación FR para transformar las unidades de medida de cada partida en $\mathrm{kg}$.

En este último punto se ha determinado el factor de relación, FR, partiendo de las densidades de los componentes, obtenidas en la literatura y en fichas técnicas de los materiales de construcción, y sus proporciones, obtenidas del BCCA, con respecto a la unidad empleada en la medición.
Se ha decidido emplear el peso en lugar del volumen como parámetro a comparar por considerarse su característica más estable y representativa a nivel de impacto medioambiental a lo largo de la ejecución de las obras.

La herramienta termina con el análisis de los resultados obtenidos, el cual permite identificar con facilidad los materiales que constituyen más del $80 \%$ del peso y, por tanto, condicionan el nivel de impacto medioambiental de la edificación.

\section{APLICACIÓN PRÁCTICA: CUANTIFICACIÓN DE LOS RECURSOS CONSUMIDOS EN EL SUBSISTEMA CONSTRUCTIVO CIMENTACIÓN}

Para aclarar lo expuesto, se ha tomado como ejemplo el subsistema constructivo cimentación de tres proyectos $\left(\mathrm{P}_{1}, \mathrm{P}_{2}\right.$ y $\left.\mathrm{P}_{3}\right)$ que se corresponden con el que hemos definido como modelo constructivo convencional español. Los resultados obtenidos en cuanto a la cuantificación del consumo de recursos se detallan en la Tabla 2, donde Qt/Sc es la cantidad total de material consumido, Qt, en el subsistema del proyecto estudiado, $\mathrm{P}_{1}$, $\mathrm{P}_{2} \mathrm{O} \mathrm{P}_{3}$, expresada en la unidad de medida empleada en la BCCA por $\mathrm{m}^{2}$ de superficie construida de edificación, Sc.

Una vez identificados los materiales en las clasificaciones establecidas es necesario transformar sus cantidades en unidades de

Tabla 2

Cuantificación de recursos. Subsistema constructivo cimentación

\begin{tabular}{|c|c|c|c|c|}
\hline \multicolumn{5}{|c|}{$\begin{array}{c}\text { CUANTIFICACIÓN DE RECURSOS } \\
\text { SUBSISTEMA CONSTRUCTIVO CIMENTACIÓN }\end{array}$} \\
\hline \multirow{2}{*}{ UD } & \multirow{2}{*}{ MATERIAL EMPLEADO } & \multicolumn{3}{|c|}{$\mathrm{Qt} / \mathrm{Sc}\left(\mathrm{UD} / \mathrm{m}^{2}\right)$} \\
\hline & & $P_{1}$ & $\mathbf{P}_{2}$ & $P_{3}$ \\
\hline $\mathrm{kg}$ & Acero & 11,7671 & 10,6563 & 11,0674 \\
\hline 1 & Aditivo & 0,3208 & 0,4024 & 0,3127 \\
\hline I & Agua & 32,2320 & 75,1775 & 37,6972 \\
\hline $\mathrm{kg}$ & Alambre de atar & 0,0544 & 0,0535 & 0,0512 \\
\hline $\mathrm{m}^{3}$ & Albero en rama & 0,0935 & 0,1440 & 0,1829 \\
\hline $\mathrm{kg}$ & Arena & 179,0935 & 179,6032 & 144,1521 \\
\hline $\mathrm{T}$ & Cemento & 0,0490 & 0,0619 & 0,0507 \\
\hline 1 & Disolvente & 0,0122 & 0,0121 & 0,0113 \\
\hline $\mathrm{kg}$ & Grava & 184,1386 & 233,0955 & 187,9391 \\
\hline $\mathrm{mu}$ & Ladrillo hueco doble $9 \mathrm{~cm}$ & 0,0008 & 0,0000 & 0,0000 \\
\hline $\mathrm{m}^{2}$ & Lamina polietileno $0,2 \mathrm{~mm}$ & 0,2701 & 0,4541 & 0,2793 \\
\hline $\mathrm{kg}$ & Pintura al clorocaucho & 0,0860 & 0,0858 & 0,0801 \\
\hline $\mathrm{m}^{3}$ & Poliestireno planchas rígidas & 0,0004 & 0,0000 & 0,0000 \\
\hline $\mathrm{kg}$ & Polvo de sílice y cuarzo & 0,9723 & 0,9697 & 0,9051 \\
\hline
\end{tabular}


Tabla 3

Valores del factor de relación FR empleado en función del material

\begin{tabular}{|l|r|}
\hline \multicolumn{1}{|c|}{ MATERIAL } & \multicolumn{1}{c|}{ FR } \\
\hline Aditivos fabricación hormigones (plastificantes y acelerantes) & $1,170 \mathrm{~kg} / \mathrm{l}$ \\
\hline Agua & $1000,000 \mathrm{~kg} / \mathrm{m}^{3}$ \\
\hline Albero en rama & $1300,000 \mathrm{~kg} / \mathrm{m}^{3}$ \\
\hline Disolvente (usado en tratamiento superficial de soleras) & $0,900 \mathrm{~kg} / \mathrm{l}$ \\
\hline Ladrillo hueco doble 9 cm (usado como encofrado perdido) & $1000,000 \mathrm{~kg} / \mathrm{m}^{3}$ \\
\hline Lámina de polietileno & $0,184 \mathrm{~kg} / \mathrm{m}^{2}$ \\
\hline Poliestireno en planchas rígidas & $12,000 \mathrm{~kg} / \mathrm{m}^{3}$ \\
\hline
\end{tabular}

Tabla 4

Materiales consumidos en el subsistema constructivo cimentación y su repercusión en la obra

\begin{tabular}{|c|c|c|c|c|c|}
\hline \multicolumn{6}{|c|}{$\begin{array}{l}\text { RECURSOS MATERIALES CONSUMIDOS EN EL SUBSISTEMA } \\
\text { CONSTRUCTIVO CIMENTACIÓN Y SU REPERCUSIÓN EN LA OBRA }\end{array}$} \\
\hline \multirow{2}{*}{ MATERIAL EMPLEADO } & \multicolumn{3}{|c|}{$\mathrm{Qt} / \mathrm{Sc}\left(\mathrm{kg} / \mathrm{m}^{2}\right)$} & \multirow{2}{*}{$R\left(\mathrm{~kg} / \mathrm{m}^{2}\right)$} & \multirow{2}{*}{$\%$} \\
\hline & $P_{1}$ & $\mathbf{P}_{2}$ & $\mathbf{P}_{3}$ & & \\
\hline Acero & 11,7571 & 10,6563 & 11,0674 & 11,1603 & 1,6727 \\
\hline Aditivo & 0,3753 & 0,4708 & 0,3658 & 0,4040 & 0,0605 \\
\hline Agua & 32,2320 & 75,1775 & 37,6972 & 48,3689 & 7,2494 \\
\hline Alambre de atar & 0,0544 & 0,0535 & 0,0512 & 0,0531 & 0,0080 \\
\hline Albero en rama & 121,5500 & 187,1829 & 237,7869 & 182,1732 & 27,3035 \\
\hline Arena & 179,0935 & 179,6032 & 144,1521 & 167,6163 & 25,1218 \\
\hline Cemento & 48,9613 & 61,8875 & 50,7441 & 53,8643 & 8,0730 \\
\hline Disolvente & 0,0109 & 0,0109 & 0,0102 & 0,0107 & 0,0016 \\
\hline Grava & 184,1386 & 233,0955 & 187,9391 & 201,7244 & 30,2338 \\
\hline Ladrillo hueco doble $9 \mathrm{~cm}$ & 2,2300 & - & - & 2,2300 & 0,3342 \\
\hline Lamina polietileno $0,2 \mathrm{~mm}$ & 0,0497 & 0,0836 & 0,0514 & 0,0616 & 0,0092 \\
\hline Pintura al clorocaucho & 0,0860 & 0,0858 & 0,0801 & 0,0840 & 0,0126 \\
\hline Poliestireno planchas rígidas & 0,0048 & - & - & 0,0048 & 0,0007 \\
\hline Polvo de sílice y cuarzo & 0,9723 & 0,9697 & 0,9051 & 0,9490 & 0,1422 \\
\hline TOTALES & 581,5160 & 749,2772 & 670,8505 & 667,2146 & 100,0000 \\
\hline
\end{tabular}

medida comparables. Para ello se aplica el factor de relación, FR, correspondiente a cada tipo de material, Tabla 3.

En la Tabla 4 se resumen los resultados obtenidos tras homogeneizar las unidades de medida de Qt/Sc en kg por $\mathrm{m}^{2}$ de superficie construida de edificación mediante la aplicación del factor de relación FR. De este modo, el nuevo Qt/Sc representa la cantidad total de material consumido en el subsistema constructivo cimentación en cada uno de los tres proyectos estudiados, $\mathrm{P}_{1}, \mathrm{P}_{2}$ y $\mathrm{P}_{3}$, expresado en $\mathrm{kg}$ por $\mathrm{m}^{2}$ de superficie construida de edificación.

El siguiente paso es determinar la repercusión de cada material. Se calcula la media ponderada, $\mathrm{R}$, del consumo de recursos en $\mathrm{kg} / \mathrm{m}^{2}$ de cada uno de los tres proyectos objeto de estudio y su correspondiente repercusión en tanto por ciento en relación con el conjunto de recursos consumidos en el subsistema cimentación. En el cálculo de la media ponderada se entiende que en caso de no consumirse el recurso en alguno de los proyectos evaluados, la media se obtiene a partir de los restantes proyectos. Así por ejemplo, en la Tabla 4 sólo el proyecto $\mathrm{P}_{1}$ emplea ladrillo hueco doble de $9 \mathrm{~cm}$ y por tanto $\mathrm{R}$ coincide con el consumo de dicho proyecto.
Finalmente, se ha obtenido la repercusión en el proceso de ejecución, considerando con valor cero aquellos recursos cuyo peso es mínimo; esto es aquéllos sin ninguna cifra entera y, en consecuencia, despreciable su afección, quedando los resultados detallados en la Tabla 5.

\section{CONCLUSIONES}

Un primer análisis de los resultados obtenidos nos permite identificar que la grava, el albero y la arena son los recursos que controlan el proceso de cimentación, representando el $82 \%$ de todo el peso de material consumido. Si nos centramos en estos tres materiales y reducimos su impacto medio ambiental estaremos reduciendo a su vez las barreras contra la sostenibilidad del subsistema cimentación. De este modo podemos concluir que la mayoría de los recursos consumidos en el subsistema constructivo cimentación, grava, albero, arena y agua, son no renovables o escasos en el medio natural (9) constituyendo el $90 \%$ de los recursos empleados, Tabla 5. De lo anterior se deduce que optimizando el consumo de materiales pétreos y agua se reduce sensiblemente el impacto medioambiental del subsistema cimentación. 
Tabla 5

Identificación de barreras en el subsistema constructivo cimentación

\begin{tabular}{|c|c|c|c|}
\hline \multicolumn{4}{|c|}{$\begin{array}{l}\begin{array}{c}\text { EVALUACIÓN DEL IMPACTO MEDIOAMBIENTAL DE LOS RECURSOS CONSUMIDOS } \\
\text { CIMENTACIÓN }\end{array} \\
\text { EN EL }\end{array}$} \\
\hline RECURSOS & \multirow{2}{*}{$\%$} & \multirow{2}{*}{$\begin{array}{c}\text { RECURSOS } \\
\text { TÓXICOS O CONTAMINANTES }\end{array}$} & \multirow{2}{*}{$\%$} \\
\hline NO RENOVABLES O ESCASOS & & & \\
\hline Grava & 30 & Cemento & 8 \\
\hline Albero & 27 & Acero & 2 \\
\hline Arena & 25 & Aditivos & 0 \\
\hline Agua & 8 & Alambre & 0 \\
\hline \multirow[t]{5}{*}{ Polvo de sílice y cuarzo } & 0 & Disolvente & 0 \\
\hline & & Pintura & 0 \\
\hline & & Ladrillo & 0 \\
\hline & & Poliestireno & 0 \\
\hline & & Lámina de polietileno & 0 \\
\hline TOTAL & 90 & TOTAL & 10 \\
\hline
\end{tabular}

Así por ejemplo, una solución de inmediata aplicación para reducir dicho impacto podría ser el reciclado de los residuos pétreos en plantas de valorización y su posterior uso como material de relleno en la misma o en otras obras o en aplicaciones tales como carriles o zanjas, Figura 3. Por otro lado, el $10 \%$ restante, coincidente con los recursos elaborados, tienen afección ambiental por su toxicidad para las personas o la contaminación del medio que produce su fabricación o puesta en obra, tal es el caso del cemento y el acero (10). De todo lo anterior se deduce que en el actual modelo constructivo convencional el $100 \%$ de los recursos empleados constituyen de una u otra forma una barrera contra la sostenibilidad provocando un impacto negativo en el medio ambiente bien por no ser renovables o bien por su toxicidad. Por lo tanto, la necesidad de buscar alternativas sostenibles con premura a dicho subsistema es manifiesta.

Una limitación del modelo, que se debe desarrollar en investigaciones y trabajos futuros, es que no cuantifica el consumo energético o las emisiones de $\mathrm{CO}_{2}$ a la atmósfera que pueden ser valores tan o más significativos desde el punto de vista de barreras contra la sostenibilidad que el parámetro elegido en el presente trabajo, que ha sido la masa de los materiales.
Asimismo, será objeto de futuros trabajos verificar estas aproximaciones y recoger todas aquellas recomendaciones, tanto relativas al proyecto como a la ejecución de la obra, que permitan definir modelos constructivos alternativos al convencional que minimicen sus consecuencias ambientales, satisfaciendo adecuadamente las demandas de construcción de una comunidad sin hipotecar las posibilidades de las generaciones futuras para disponer de los recursos adecuados y de un medio ambiente de calidad.

\section{AGRADECIMIENTOS}

Gracias al proyecto de investigación SUSPURPOL (Sustainable Purchasing and Planning Policy Blueprint Project) perteneciente al programa europeo INTERREG III, ha podido intercambiarse información en relación con las barreras contra la sostenibilidad entre los países miembros del proyecto: Inglaterra, Polonia y España; lo que ha sido de gran utilidad para corroborar los resultados obtenidos en la investigación.

Los trabajos relativos a la identificación del modelo constructivo convencional y cuantificación de recursos consumidos constituyen parte de la tesis doctoral que actualmente desarrolla la profesora $\mathrm{M}^{\mathrm{a}}$ del Pilar Mercader.
3. Aplicaciones de residuos pétreos reciclados

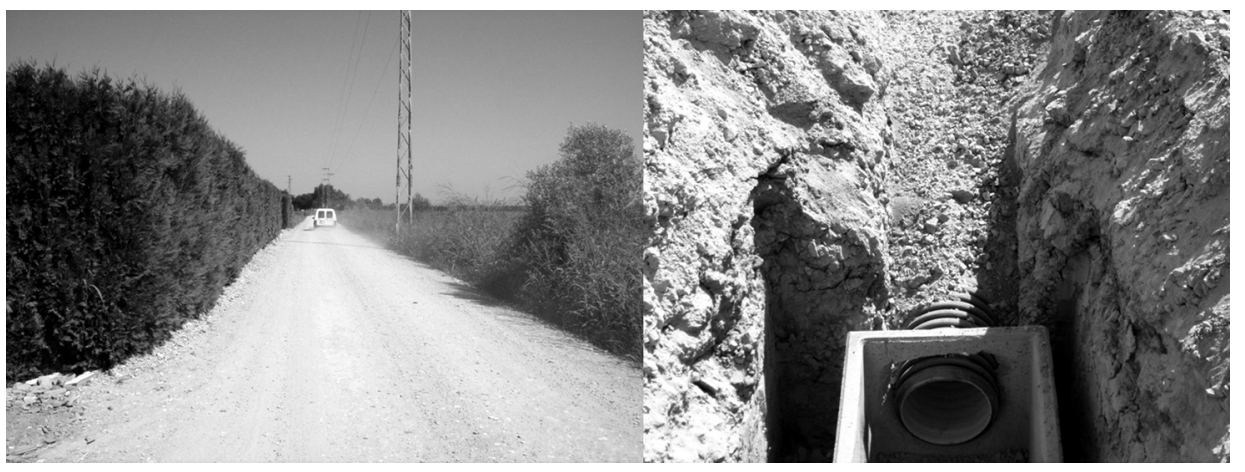




\section{BIBLIOGRAFÍA}

(1) Baño Nieva, A. y Vigil-Escalera del Pozo, A.: Guía de construcción sostenible. p. 9, Instituto Sindical de Trabajo, Ambiente y Salud (ISTAS). España, 2005.

(2) Solanas, T. y Herreros, J.: Vivienda y sostenibilidad en España. Vol. 2: Colectiva. pp. 15-16, Editorial Gustavo Gili. Barcelona, 2008.

(3) Ramírez de Arellano Agudo, Llatas-Oliver, A. C., García-Torres, I., Linares-Romero, P., GarcíaCaraballo, E.I., Escobar García, M., Carnerero Moya, M. y Hernández Juárez, R.: Retirada Selectiva de Residuos: Modelo de Presupuestación. p.5, Fundación Aparejadores. Sevilla, 2002.

(4) Cuchí, A., Wadel, G., López, F. y Sagrera, A.: Guía de la eficiencia energética para administradores de fincas. pp. 10-11, Fundación Gas Natural. España, 2007.

(5) Dirección General de Programación Económica y Presupuestaria. Índice de precios de las viviendas 2000: estadística de precio medio del $\mathrm{m}^{2}$ : datos obtenidos de las tasaciones hipotecarias. Ministerio de Fomento. Madrid, 2001.

(6) Dirección General de Programación Económica y Presupuestaria. Licitación oficial en construcción,2001. Ministerio de Fomento. Madrid, 2002.

(7) Dirección General de Programación Económica y Presupuestaria. Edificación y Vivienda: 19982003. Ministerio de Fomento. Madrid, 2004.

(8) Consejería de Vivienda y Ordenación del Territorio. Base de Costes de la Construcción de Andalucía 2008. Junta de Andalucía, 2008. Página web:

http: www.juntadeandalucia.es/viviendayordenaciondelterritorio/www/jsp/estatica.jsp?pma=0\&ct=$1 \&$ pmsa=0\&e=biblioteca_archivos/../planificacion/publicaciones/banco_precios_construccion/ bcca08/bcca08.html

(9) Spiegel, R. y Meadows, D.: Green Building Materials. John Wiley \& Sons, Inc. EEUU, 1999.

(10) Sustainable Buildings Industry Council. Green building guidelines: Meeting the demand for lowenergy, resource efficient homes. Sustainable Buildings Industry Council. EEUU, 2004. 